\title{
FORMES DE MULTIPLICATION DES EIMERIA \\ RENCONTRÉES CHEZ UN JEUNE TATOU
}

\author{
Par A. CARINI
}

Dans une note précédente, relative à deux nouvelles Eimeria de l'intestin d'un jeune tatou(1), nous avons décrit les oocystes de ces coccidies. Comme on peut le voir d'après les dessins et la description que nous avons donnés, la morphologie générale des deux types d'oocystes est d'une ressemblance remarquable, ce qui fait penser qu'il s'agit peut-être d'une même espèce, pouvant présenter des oocystes de dimensions variables. Toutefois, si l'on considère les autres caractères différentiels, et le fait que l'on ne trouve pas de formes intermédiaires, nous croyons que l'on est, du moins provisoirement, autorisé à considérer les deux formes d'oocystes comme appartenant à deux espèces différentes.

$$
* *
$$

En examinant les coupes de différents segments de l'intestin du tatou parasité, nous avons rencontré quelques formes de multiplication sexuée, qui appartiennent vraisemblablement au cycle évolutif d'une des deux Eimeria décrites précédemment.

Dans nos préparations on ne trouvait plus de formes de multiplication schizogonique. Les macrogamètes ne sont pas nombreux, mais on peut en trouver assez facilement, tandis que les microgamétocytes sont extrêmement rares, et il faut chercher très longtemps pour en observer quelques-uns.

Les macrogamètes sont parfois isolés, parfois réunis en groupes de forme arrondie, composés de 8 à 12 individus. Les macrogamètes isolés se rencontrent de préférence dans l'intestin grêle, vers le sommet des villosités, tandis que les groupes siègent dans le gros intestin et occupent généralement le fond d'une glandule, qui est dilatée par la présence des parasites (fig. 1).

Les macrogamètes sont ronds, d'un diamètre de 18 à $20 \mu$; au

(1) Annales de Parasitologie, XI, 1933, p. 469.

Annales de Parasitologie, T. XII, ${ }^{\circ} 1 .-1^{\text {er }}$ janvier 1934, p. 58-60. 
centre, il y a un noyau de forme étoilée, entouré de vaeuoles claires. Dans le noyau on aperçoit un petit caryosome.

A mesure que les macrogamètes mûrissent, il se forme dans le

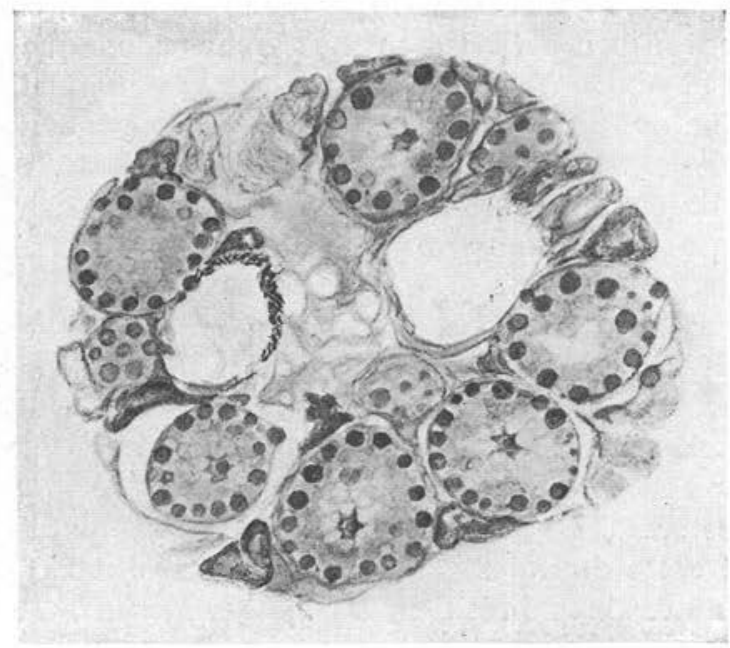

F1G. 1. - Groupe de macrogamètes d'Eimeria observées dans le gros intestin d'un jeune tatou, $\times 850$.

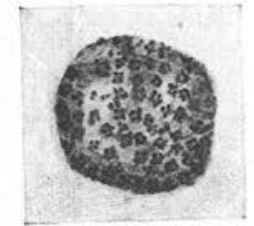

FIG. 2. - Microgamêtocyte d'Eimeria du tatou, $\times 1.800$.

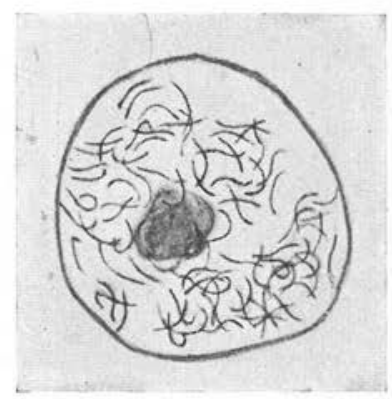

FIG. 3. - Microgamètes mûrs d'Eimeria du tatou, $\times 1.800$. On distingue au centre le corps résiduel plus ou moins vacuolisé.

protoplasme de nombreuses granulations sphériques, homogènes, de dimensions assez variables, généralement d'un diamètre de 2 à $3 \mu$. Ces granulations ont tendance à se disposer à la périphérie de 
la cellule et, dans les préparations colorées par l'hématoxylineéosine, elles apparaissent intensément colorées en rouge. Dans chaque macrogamète on peut compter plus de 50 de ces granulations.

Les microgamétocytes sont, comme nous avons dit, extrêmement rares ; ils ont à peu près les mêmes dimensions que les macrogamètes. Le noyau se divise en un grand nombre de petites masses de chromatine qui peu à peu se disposent à la périphérie (fig. 2) et donnent naissance aux microgamètes qui, lorsqu'ils sont mûrs, deviennent libres sous forme de petits filaments. Dans la partie centrale des microgamétocytes mûrs, on voit le corps résiduel plus ou moins vacuolisé (fig. 3 ).

Nous croyons que ces formes de multiplication appartiennent au cycle évolutif d'une des deux Eimeria, que nous avons précédemment décrites, mais nous ne pouvons pas dire exactement à laquelle. Nous espérons rencontrer d'autres tatous parasités et pouvoir compléter nos recherches.

Les formes sexuées que nous avons rencontrées paraissent absolument différentés de celles vues par Cunha et Muniz (1) dans l'intestin d'un autre tatou brésilien, Dasypus sexcintus.

(1) DA Cunha (A. M.) et Muniz (J.). - Sur un nouveau sporozoaire, parasite du tatou. C.R. Soc. biol., XCVIII, 1927, p. 624.

Laboratorio paulista de Biologia, Sâo Paulo (Brésil). 